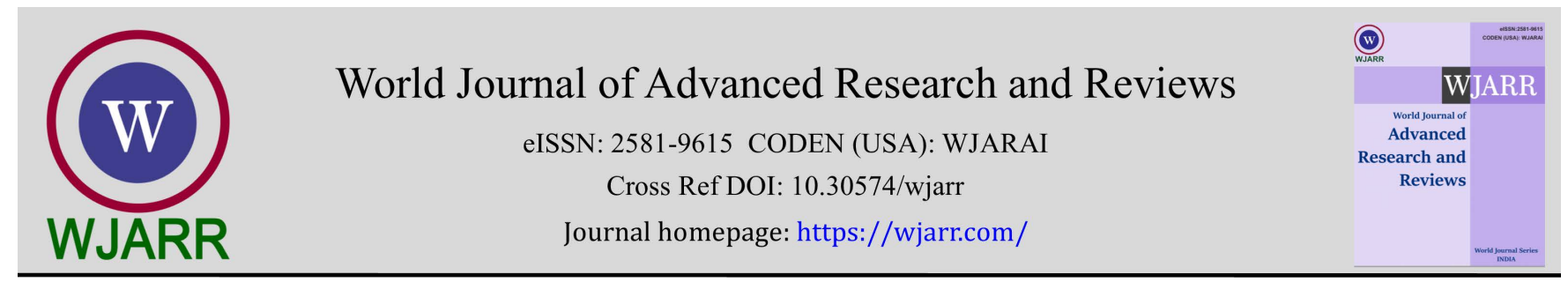

(REVIEW ARTICLE)

Check for updates

\title{
Premature rupture of membranes
}

\author{
Sefik Gokce * and Dilsad Herkiloglu \\ Department of Obstetrics and Gynecology, Yeni Yüzyıl University Private Gaziosmanpaşa Hospital, Gaziosmanpaşa, \\ Istanbul/Turkey.
}

World Journal of Advanced Research and Reviews, 2021, 11(03), 363-370

Publication history: Received on 18 August 2021; revised on 25 September 2021; accepted on 27 September 2021

Article DOI: https://doi.org/10.30574/wjarr.2021.11.3.0393

\begin{abstract}
Prenatal (premature) rupture of membranes (PROM) is defined as rupture of fetal membranes before the onset of labor or regular uterine contractions. PROM can lead to serious complications such as uterine cavity infection, umbilical cord compression, oligohydramnios, fetal malpresentation, umbilical cord prolapse, preterm delivery, fetal asphyxia and death. Initial evaluation of all term pregnancies with suspected PROM should include confirmation of membrane rupture and assessment of maternal and fetal well-being. Immediate initiation of labor is recommended in term pregnant women with PROM. Compared with expectant management, induction of labor is associated with a reduction in maternal and possibly neonatal infection and lower treatment costs without an increase in cesarean delivery. Induction with oxytocin is recommended. Oxytocin is as effective as prostaglandins, easier to titrate and may be less expensive depending on the preparation. Balloon catheter use is not recommended for cervical ripening in PROM.
\end{abstract}

Keywords: Premature rupture of membranes; PROM; Active management; Expectant management; Preterm delivery

\section{Introduction}

Prenatal (premature) rupture of membranes (PROM) is defined as rupture of fetal membranes before the onset of labor or regular uterine contractions. It can occur at term ( $\geq 37$ weeks of gestation) or preterm $(<37$ weeks of gestation). It is called PPROM in preterm. Second trimester PROM is typically efers to PPROM at 16th-26th weeks of gestation; this is a definition that varies slightly among researchers. The frequencies of term, preterm and second trimester PROM are seen in approximately $8 \%, 3 \%$ and $<1 \%$ of pregnancies, respectively. It is not fully understood why the membranes rupture at term and the different etiologies that lead to membrane rupture before labor instead of intrapartum [1,2].

\section{Prevalence}

PROM occurs in about 3\% of pregnancies:

- in approximately $0.5 \%$ of pregnancies of $<27$ weeks,

- $\quad$ in $1 \%$ of pregnancies at 27-34 weeks,

- $\quad$ in $1 \%$ of pregnancies at 34-37 weeks [1-3].

\footnotetext{
${ }^{*}$ Corresponding author: Şefik Gökçe

Department of Obstetrics and Gynecology, Yeni Yüzyıl University Private Gaziosmanpaşa Hospital, Gaziosmanpaşa, Istanbul/Turkey. Copyright @ 2021 Author(s) retain the copyright of this article. This article is published under the terms of the Creative Commons Attribution Liscense 4.0.
} 


\section{Pathogenesis}

The pathogenesis of premature rupture of membranes is not fully understood. The strength and integrity of fetal membranes is due to extracellular membrane proteins, including collagens, fibronectin, and laminin. Matrix metalloproteases (MMPs) decrease membrane strength by increasing collagen degradation. Tissue inhibitors of MMPs bind to MMPs and help maintain membrane integrity by inhibiting MMP-related proteolysis. Various pathological events (eg, subclinical or overt infection, inflammation, mechanical stress, bleeding) can disrupt this and other homeostatic processes and initiate a series of biochemical changes that result in PROM [4].

\section{Risk Factors}

Many factors such as maternal physiological, genetic and environmental predispose to the development of PROM. These risk factors are similar to those that cause preterm labor, but most patients do not have identifiable risk factors. History of PROM in a previous pregnancy, genital tract infection, antepartum hemorrhage, and smoking are particularly strongly associated with PROM and are discussed below [5].

\subsection{History of PROM}

Studies have reported that a history of PROM is a high-risk factor for recurrence [5].

\subsection{Genital Tract Infection}

Genital tract infection is the most common identifiable risk factor for PROM. There are three important epidemiological data on this subject:

- The fact that women with PROM are significantly more likely to have pathogenic microorganisms in the amniotic fluid than women with intact membranes.

- Histological chorioamnionitis rate of women with PROM.

- The fact that the prevalence of PROM is significantly higher in women with certain lower genital tract infections (especially bacterial vaginosis) than in uninfected women.

Most of the microorganisms that colonize the lower genital tract have the capacity to produce phospholipase, which can stimulate the production of prostaglandins, thereby leading to the initiation of uterine contractions. In addition, the host's immune response to bacterial invasion of the endocervix and/or fetal membranes leads to the production of multiple inflammatory mediators that can cause localized weakening of fetal membranes and result in PROM. Genetic regulation of the host's immune and inflammatory responses appears to play a role in susceptibility and response to PROM-associated infections [5,6].

\subsection{Antepartum Bleeding}

Vaginal bleeding in the first trimester is associated with a small but statistically significant increase in the risk of PROM. Antepartum hemorrhage seen in more than one trimester increases the risk of PROM three to seven times. In the case of bleeding, the development of PROM may be associated with a high decidual concentration of tissue factor (also known as factor III). In addition to hemostatic properties, contractions can initiate as a result of binding of thrombin to decidual protease-activated receptors (PAR 1 and 3), which increase the expression of membrane-disrupting proteases such as matrix metalloproteases $[5,6]$.

\subsection{Smoking}

The risk of PROM among smokers is two to four times higher than among nonsmokers. The mechanism of PROM association with smoking is unclear. In addition, several genetic polymorphisms of genes related to infection, inflammation and collagen degradation, as well as polyhydramnios and acute trauma, are reported to be potential risk factors for PROM [5,6]. 


\section{Clinical features and laboratory findings}

\subsection{Symptoms}

Sudden clear or pale yellow fluid discharges from the vagina. Some patients complain of leaking only small amounts of fluid continuously or intermittently. Others describe an abnormal feeling of wetness in the vagina or perineum.

\subsection{Physical examination}

For women who are not actively giving birth, examination of the cervix and vagina should be performed using a sterile speculum. Digital examination should be avoided as it may shorten the delay time (i.e. the time from PROM to delivery) and increase the risk of intrauterine infection. Direct observation of amniotic fluid leaking from the cervical os and ponding in the vaginal dome are pathognomonic for PROM. If the amniotic fluid is not immediately visible, the woman may be asked to push the fundus, Valsalva, or cough to cause the amniotic fluid to leak from the cervical os. The cervix may appear dilated and/or effaced, and rarely a prolapse of a fetal part or umbilical cord may occur.

\subsection{Ultrasonography}

Most patients have oligohydramnios (ie, less amniotic fluid volume than expected for gestational age). The criteria for oligohydramnios differ slightly among sonographers, but can be defined as a maximum vertical pocket (MVP) of amniotic fluid of $<2 \mathrm{~cm}$ in depth or amniotic fluid index (AFI) of $\leq 5 \mathrm{~cm}$ (some use $2 \mathrm{~cm}$ and $<5 \mathrm{~cm}$, respectively).

\subsection{Laboratory}

Hematology and biochemistry tests are normal in the absence of infection or other complications of pregnancy. Laboratory tests to identify amniotic fluid are described below [5,7].

\section{Clinical Follow-Up}

The time from PROM to delivery is inversely proportional to the gestational age and is shorter in cases with residual oligohydramnios [8].

Table 1 Pregnancy complications associated with premature rupture of membranes (PROM)

\begin{tabular}{|c|c|c|}
\hline Pregnancy complication & Possible consequences for newborns & Possible maternal consequences \\
\hline uterine cavity infection & $\begin{array}{l}\text { Neonatal sepsis } \\
\text { Long-term neurodevelopmental } \\
\text { abnormalities, especially cerebral palsy }\end{array}$ & $\begin{array}{l}\text { Chorioamnionitis } \\
\text { postpartum endometritis } \\
\text { Septicemia }\end{array}$ \\
\hline $\begin{array}{l}\text { Umbilical } \\
\text { compression }\end{array}$ & Fetal asphyxia and death & Cesarean delivery \\
\hline Oligohydramnios & $\begin{array}{l}\text { Limb restriction deformities and } \\
\text { pulmonary hypoplasia (primarily } \\
\text { severe oligohydramnios from early to } \\
\text { mid second trimester). These } \\
\text { complications are rare when membrane } \\
\text { rupture occurs after } 23 \text { weeks. }\end{array}$ & \\
\hline Fetal malpresentation & & Cesarean delivery \\
\hline Umbilical cord prolapse & Fetal asphyxia and death & Cesarean delivery \\
\hline Fetal asphyxia and death & Cesarean delivery & Coagulopathy \\
\hline Preterm delivery & $\begin{array}{l}\text { Morbidity of prematurity, including } \\
\text { respiratory } \\
\text { intraventricular abnormalities, } \\
\text { necrotizing enterocolitis, retinopathy of } \\
\text { prematurity, patent ductus arteriosus }\end{array}$ & \\
\hline
\end{tabular}


However, most pregnancies with PROM are born within one week of membrane rupture. Chorioamnionitis has been reported in approximately $60 \%$ of cases and has been seen as a common cause of initiation or initiation of spontaneous labor [9]. The risk of clinical chorioamnionitis increases twofold in cases with residual oligohydramnios [8]. Ablation of the placenta occurs in $2-5 \%$ of pregnancies complicated by PROM. The risk increases seven to nine times in PROM pregnancies complicated by intrauterine infection or oligohydramnios. Ablation of the placenta may be the precipitating event for PROM or a consequence thereof. Cessation of fluid leakage is rare, except in women with PROM due to amniocentesis. Closure of membranes is associated with a more favorable prognosis. Fetus and newborn are at higher risk of PROM-related morbidity and mortality than the mother (Table 1) [8-10].

Fetal malpresentation is common given the frequent occurrence of preterm gestational age and low amniotic fluid volume. The risk of cord prolapse is particularly high in both non-cephalic fetal presentation and PROM. Noncephalic presentation may also increase the risk of ablatio placentae, infection, and fetal death. In the absence of spontaneous labor or if complications (chorioamnionitis, ablatio placenta, cord prolapse) that will trigger delivery occur, labor induction is generally recommended for pregnancies with PROM at $\geq 34$ weeks [8-11].

\section{Initial Evaluation Of Prom}

Pregnant women with PROM should be evaluated by the clinician as soon as possible, and it should be comprehensively considered whether labor has begun and whether to follow-up at home. The most accurate approach for this is rapid assessment to confirm rupture of membranes, determine fetal position, assess maternal and fetal status, and discuss options for further management.

- The diagnosis of PROM is based on the patient's specific history (eg, vaginal fluid leakage) and speculum examination confirming that fluid is draining from the cervical os. If the diagnosis is uncertain, a diagnostic test is performed on fluid collected in the posterior fornix. However, digital cervicovaginal examination should be avoided before delivery as it is associated with an increased risk of intrauterine infection.

- $\quad$ Fetal status is evaluated with non-stress test (NST).

- Fetal position is determined by transabdominal physical examination (Leopold's maneuvers) and/or ultrasound examination (ultrasound dominates the modern assessment of fetal presentation in the PROM setting).

- Maternal evaluation includes evaluation of contractions and signs of infection (eg, fever, fetal tachycardia) and review of prenatal records and current maternal status for medical and obstetric complications that affect decision-making regarding timing of delivery $[12,13]$.

\section{Management of prom}

The key decision in uncomplicated PROM management is whether to initiate labor or take a waiting approach. We recommend immediate delivery for women with PROM at term. Labor is initiated as soon as possible unless there are contraindications for labor or vaginal delivery. If there is a contraindication, cesarean delivery is performed as soon as possible $[12,13]$.

Our approach is primarily based on our concerns about pregnancy management and the increased risk of maternal and neonatal infection, as well as our patients' general preferences for rapid delivery. Immediate intervention also reduces the risk of other serious but less common complications such as cord prolapse or injury and was the most cost-effective approach in one model [13-14]. However, induction labor tends to be longer than spontaneous labor. For women who refuse emergency response, it is recommended to set a time limit for expected management in the form of joint decision making. Signs of infection or other pregnancy complications are an indication for termination of waiting management and delivery by the most appropriate method for the clinical situation [14-16]. The American College of Obstetricians and Gynecologists recommends giving birth at $\geq 37+0$ weeks for women with PROM, but states that a short wait management may also be recommended.

\subsection{Active Management}

\subsubsection{Oxytocin induction}

For women without contraindications for labor and vaginal delivery, induction with oxytocin is recommended without cervical ripening prior to induction. Oxytocin is easier to use than prostaglandins and may be cheaper depending on the 
prostaglandin preparation chosen. Meta-analyzes of randomized studies have not shown a clear benefit from the initial use of any prostaglandin instead of oxytocin in women with PROM, including those with an unfavorable cervix, but this practice for patients with an unfavorable cervix is controversial [16-18].

\subsection{Alternatives for Women with an Unfavorable Cervix}

\subsubsection{Misoprostol or prostaglandin E2}

Although pre-induction cervical ripening is not performed in term PROM, some clinicians may choose to use prostaglandin E1 or E2 after considering the advantages and disadvantages. Misoprostol (prostaglandin E1) is an effective method of induction of labor. It is also inexpensive, readily available, and stable at room temperature.

\subsubsection{Vaginal administration}

According to the guidelines of the American College of Obstetricians and Gynecologists, an initial dose of $25 \mathrm{mcg}$ misoprostol is applied to the posterior fornix. It is repeated every 3-6 hours depending on the maternal and fetal response. In patients with no cervical changes three hours after administration of a dose and only minimal uterine activity, a three-hour interval between doses is appropriate.

\subsubsection{Oral administration}

The World Health Organization recommends administration of $25 \mathrm{mcg}$ misoprostol every two hours. Regardless of the route of administration, the dose of misoprostol is usually repeated until the cervix is fit, labor pains occur, or until five doses have been administered. Prostaglandin E2 preparations are administered according to the manufacturer's instructions (package leaflet).

\subsubsection{Balloon catheter}

It is recommended not to use a balloon catheter for cervical ripening in the case of PROM. No reliable data are available to support safety and efficacy in these patients. There is evidence of an increased risk of chorioamnionitis. In this case, both oxytocin and prostaglandins are safe and effective alternatives. A randomized trial ( $\mathrm{n}=128$ women) of patients undergoing cervical ripening with balloon catheter and at term or recent PROM observed no difference in time from induction to delivery in patients induced by oxytocin alone versus concomitant balloon catheter+oxytocin, with a trend towards increased risk of maternal infection (chorioamnionitis $10 \%$ vs. 5\%) [18-22].

\section{Follow-Up of the Patients}

After assessing the risks and benefits of induction in treatment for patients, women with uncomplicated pregnancies and avoiding emergency intervention can be followed up for a reasonable period of time. However, there should be no contraindications for labor and vaginal delivery in these patients. In addition, the fetal test should be normal and there should be no indication of clinical chorioamnionitis or other medical or obstetric complications that may increase maternal/fetal risk if delivery is delayed [22-24]

Women with meconium-stained amniotic fluid should not be followed up for PROM. Meconium-stained amniotic fluid, clinical chorioamnionitis, and positive amniotic fluid cultures have been associated with impaired intrapartum fetal heart rate movements and an increased risk of meconium aspiration syndrome. However, there is no evidence that initiating immediate labor will reduce the risk of these complications; therefore, if this is the approach chosen by the patient and the antepartum fetal assessment is otherwise reassuring (NST without variable deceleration), it is believed that lightly stained amniotic fluid with meconium is not a strong barrier to pregnancy follow-up. In some of these cases, the meconium-like staining is actually a sign that may result from decidual bleeding. Conversely, we believe that induction and continuous fetal monitoring is a prudent approach when meconium is thick [22-27].

\subsection{Duration of expectant management}

There are no strong data to base a recommendation for the maximum duration of expectant management in women with advanced labor who do not have pregnancy complications. In studies that limited expectant management to 96 hours after rupture, the risk of chorioamnionitis was found to increase significantly after 24 hours. As a result of these studies, 24 hours was considered to be a reasonable limit. However, expecting longer will increase the number of women whose labor will start spontaneously [22-24]. 


\subsection{Expectant management; in the hospital or at home?}

It is recommended that women with PROM be hospitalized. Studies have shown that women who are sent home are more likely to develop clinical chorioamnionitis. In addition, it has been observed that the newborns of pregnant women followed at home have a higher risk of infection. In addition, nulliparous patients who are administered expect-at-home management may need to take antibiotics before delivery. Another risk in patients followed up at home is the possibility of rapid labor and delivery before the patient goes to the hospital [24-28].

The following criteria are recommended for patients who request home follow-up until labor or a complication occurs:

- If there is cephalic presentation

- If there is no uterine cavity infection

- If there is a reassuring fetal heart rate

- If there is at least $2 \times 2 \mathrm{~cm}$ amniotic fluid pocket on ultrasound

- If the patient is reliable

- If home assistance is available

- If there is reliable transportation

- If the house can be reached within 20 minutes from the hospital

- If there is the ability to check pulse and temperature every six hours, provided that their clinician is informed by parameters (pulse $>100$ beats per minute, temperature $\geq 38{ }^{\circ} \mathrm{C}$ )

- If the daily fetal beats are good

- If daily NST and leukocyte count can be obtained [22-29].

\subsection{Antibiotic prophylaxis}

As mentioned above, it was an approach to minimize the risk of infection, immediate induction of labor and use of intrapartum group B Streptococcus (GBS) antibiotic prophylaxis at the time specified by standard guidelines is recommended rather than follow-up with (or without) broad-spectrum antibiotic prophylaxis. Considering the potential side effects of antibiotic use, the potential for the development of resistant microorganisms, and the low risk of maternal infection in untreated patients, prophylactic antibiotic use should be avoided for PROM at or near term. There is no strong evidence of a maternal or neonatal benefit of prophylactic antibiotic use for PROM at or near term. It has been reported that antibiotic prophylaxis can reduce the risk of maternal infection in 37\% of pregnancies where the delay is longer than 12 hours. However, it was stated that there was no statistical improvement in terms of neonatal outcomes. No data were available on the possible harms of this treatment. The relative risks and benefits of antibiotic prophylaxis in the preterm period are different. Although the available data are limited, they support the use of antibiotic prophylaxis in preterm PROM because it prolongs the "delay time in labor" which is not a target in term PROM but desired in preterm pregnancies.

\subsection{Group B Streptococcus colonization}

Induction is recommended for pregnant women who are positive for GBS. In these patients, induction with oxytocin was associated with a lower rate of neonatal infection than waiting management ( $2.5 \% \mathrm{vs}$. $8 \%)$. In women with positive GBS cultures, regardless of induction or standby management, maternal antibiotic prophylaxis should be initiated promptly to reduce the risk of disease. If GBS status is unknown, the decision to initiate antibiotics is based on standard risk factor assessment.

\subsection{Maternal and fetal monitoring}

There is no standard for maternal-fetal monitoring in PROM at term with expectant management. There is consensus that digital (digital) vaginal examination and vaginal intercourse should be avoided to reduce the risk of uterine cavity infection. Women who will undergo standby management should check their temperature twice daily and report when their temperature is $\geq 37.5^{\circ} \mathrm{C}$, monitor and report changes in the color or odor of vaginal discharge, and inform their clinician if any complications develop. Changes in fetal heart rate should be kept in mind as an important finding, as it may first suggest cord prolapse or cord compression.

\subsection{Complete blood count}

Leukocytosis in pregnant women is a nonspecific finding and may be associated with inflammation apart from infection. Impending chorioamnionitis should be suspected when leukocytosis occurs with other premonitory signs or symptoms 
of infection, or when the white cell count is elevated, unless there is a marked elevation of the white blood cell count (more than 20,000) or a significant leftward shift.

\subsection{Indications for delivery}

Patient follow-up is terminated when the time limit defined by the clinician and patient is reached or any standard indication for delivery develops (Suspected chorioamnionitis, non-reactive NST, low biophysical profile score, preeclampsia) [27-29].

\section{Conclusion}

- Prenatal (premature) rupture of membranes (PROM) refers to the rupture of fetal membranes before the onset of labor or regular uterine contractions.

- Initial evaluation of all term pregnancies with suspected PROM should include confirmation of membrane rupture and assessment of maternal and fetal well-being. The need for group B streptococcal chemoprophylaxis is also decided.

- Immediate initiation of labor is recommended in term pregnant women with PROM. Compared with waiting management, induction of labor is associated with a reduction in maternal and possibly neonatal infection and lower treatment costs without an increase in cesarean delivery.

- Induction with oxytocin is recommended (Grade 2B). Oxytocin is as effective as prostaglandins, easier to titrate and may be less expensive depending on the preparation. Balloon catheter use is not recommended for cervical ripening in PROM.

\section{Compliance with ethical standards}

\section{Disclosure of conflict of interest}

None declared.

\section{References}

[1] Coates D, Makris A, Catling C, Henry A, Scarf V, Watts N, et al. A systematic scoping review of clinical indications for induction of labour. PLoS One. 2020; 15(1): e0228196.

[2] Committee on Practice Bulletins-Obstetrics. ACOG Practice Bulletin No. 188: Prelabor Rupture of Membranes. Obstet Gynecol. 2018; 131(1): e1-e14.

[3] van der Heyden JL. Preterm prelabor rupture of membranes: different gestational ages, different problems. Maastricht University. 2014.

[4] Kumar D, Moore RM, Mercer BM, Mansour JM, Redline RW, Moore JJ. The physiology of fetal membrane weakening and rupture: Insights gained from the determination of physical properties revisited. Placenta. 2016; 42: 59-73.

[5] Duff P, Lockwood CJ, Barss VA. Preterm prelabor rupture of membranes: Clinical manifestations and diagnosis. 2020.

[6] Goldenberg RL, Culhane JF, Iams JD, Romero R. Epidemiology and causes of preterm birth. Lancet. 2008; 371(9606): 75-84.

[7] Wong LF, Holmgren CM, Silver RM, Varner MW, Manuck TA. Outcomes of expectantly managed pregnancies with multiple gestations and preterm premature rupture of membranes prior to 26 weeks. Am J Obstet Gynecol. 2015; 212(2): 215.e1-9.

[8] Pergialiotis V, Bellos I, Fanaki M, Antsaklis A, Loutradis D, Daskalakis G. The impact of residual oligohydramnios following preterm premature rupture of membranes on adverse pregnancy outcomes: a meta-analysis. Am J Obstet Gynecol. 2020; 222(6): 628-630.

[9] Beydoun SN, Yasin SY. Premature rupture of the membranes before 28 weeks: conservative management. Am J Obstet Gynecol. 1986; 155(3): 471-479. 
[10] Ananth CV, Oyelese Y, Srinivas N, Yeo L, Vintzileos AM. Preterm premature rupture of membranes, intrauterine infection, and oligohydramnios: risk factors for placental abruption. Obstet Gynecol. 2004; 104(1): 71-77.

[11] Lewis DF, Robichaux AG, Jaekle RK, Salas A, Canzoneri BJ, Horton K, et al. Expectant management of preterm premature rupture of membranes and nonvertex presentation: what are the risks? Am J Obstet Gynecol. 2007; 196(6): 566.e1-566e5

[12] Mozurkewich E. Prelabor rupture of membranes at term: induction techniques. Clin Obstet Gynecol. 2006; 49(3): 672-683.

[13] American College of Obstetricians and Gynecologists' Committee on Practice Bulletins-Obstetrics . Prelabor Rupture of Membranes: ACOG Practice Bulletin. 2019; 135 (3): e80-e97.

[14] Kehl S, Weiss C, Dammer U, Baier F, Faschingbauer F, Beckmann MW, et al. Effect of Premature Rupture of Membranes on Induction of Labor: A Historical Cohort Study. Geburtshilfe Frauenheilkd. 2017; 77(11): 11741181.

[15] Saccone G, Della Corte L, Maruotti GM, Quist-Nelson J, Raffone A, De Vivo V, et al. Induction of labor at full-term in pregnant women with uncomplicated singleton pregnancy: A systematic review and meta-analysis of randomized trials. Acta Obstet Gynecol Scand. 2019; 98(8): 958-966.

[16] Hofmeyr GJ, Gülmezoglu AM, Pileggi C. Vaginal misoprostol for cervical ripening and induction of labour. Cochrane Database Syst Rev. 2010; (10): CD000941.

[17] Induction of labour with a favourable cervix and/or pre-labour rupture of membranes. Best Pract Res Clin Obstet Gynaecol. 2003; 17(5): 795-809.

[18] Hidalgo-Lopezosa P, Hidalgo-Maestre M, Rodríguez-Borrego MA. Labor stimulation with oxytocin: effects on obstetrical and neonatal outcomes. Rev Lat Am Enfermagem. 2016; 24: e2744.

[19] Güngördük K, Asicioglu O, Besimoglu B, Güngördük OC, Yildirm G, Ark C, et al. Labor induction in term premature rupture of membranes: comparison between oxytocin and dinoprostone followed 6 hours later by oxytocin. Am J Obstet Gynecol. 2012; 206(1): 60.e1-e8.

[20] ACOG Practice Bulletin No. 107: Induction of labor. Obstet Gynecol. 2009; 114(2 Pt 1): 386-397.

[21] Tang J, Kapp N, Dragoman M, de Souza JP. WHO recommendations for misoprostol use for obstetric and gynecologic indications. Int J Gynaecol Obstet. 2013; 121(2): 186-189.

[22] Marconi AM. Recent advances in the induction of labor. F1000Res. 2019; 8.

[23] Amorosa JMH, Stone J, Factor SH, Booker W, Newland M, Bianco A. A randomized trial of Foley Bulb for Labor Induction in Premature Rupture of Membranes in Nulliparas (FLIP). Am J Obstet Gynecol. 2017; 217(3): 360.e1360.e7.

[24] Romero R, Yoon BH, Chaemsaithong P, Cortez J, Park CW, Gonzalez R, et al. Bacteria and endotoxin in meconiumstained amniotic fluid at term: could intra-amniotic infection cause meconium passage? J Matern Fetal Neonatal Med. 2014; 27(8): 775-788.

[25] Tita AT, Andrews WW. Diagnosis and management of clinical chorioamnionitis. Clin Perinatol. 2010; 37(2): 339354.

[26] Berger R, Abele H, Bahlmann F, Bedei I, Doubek K, Felderhoff-Müser U, et al. Prevention and Therapy of Preterm Birth. Guideline of the DGGG, OEGGG and SGGG (S2k Level, AWMF Registry Number 015/025, February 2019) Part 2 with Recommendations on the Tertiary Prevention of Preterm Birth and the Management of Preterm Premature Rupture of Membranes. Geburtshilfe Frauenheilkd. 2019; 79(8): 813-833.

[27] Ellestad SC, Swamy GK, Sinclair T, James AH, Heine RP, Murtha AP. Preterm premature rupture of membrane management--inpatient versus outpatient: a retrospective review. Am J Perinatol. 2008; 25(1): 69-73.

[28] Saccone G, Berghella V. Antibiotic prophylaxis for term or near-term premature rupture of membranes: metaanalysis of randomized trials. Am J Obstet Gynecol. 2015; 212(5): 627.e1-627.e9.

[29] Wojcieszek AM, Stock OM, Flenady V. Antibiotics for prelabour rupture of membranes at or near term. Cochrane Database Syst Rev. 2014; (10): CD001807. 